TITLE:

\title{
Lexicographically optimal base of a submodular system with respect to a weight vector
}

$\operatorname{AUTHOR}(\mathrm{S})$ :

Iwamura, Kakuzo

\section{CITATION:}

Iwamura, Kakuzo. Lexicographically optimal base of a submodular system with respect to a weight vector. 数理解析研究所講究録 1990, 731: 166-176

\section{ISSUE DATE:}

1990-10

URL:

http://hdl.handle.net/2433/101969

RIGHT: 
Lexicographically optimal base of a submodular system with respect to a weight rector

\author{
by \\ Kakuzo Iwamura (岩村党三) \\ Dept. of Mathematics (土成西大学) \\ Josai Univ. 数学教室
}

\begin{abstract}
We show the existence of a lexicographically optimal base of a submodular system with respect to a weight vector. We also show a greedy procedure to get it through on algebraic consideration.
\end{abstract}

\title{
1. Introduction
}

Submodular system has been developed by S. Fujlshige (1978-1987]. He posed an algorithm to get a lexicographically optimal base of a polymatroid with respect to a weight vector through geometric consideration [1980). We have shown that the same results hold for a submodular system with $f(A)>$ $0(\theta \neq A \in \mathcal{D})$ and have presented a greedy procedure in an algebraic way [1987]. In response to our work and to questions proposed by the author, $S$. Fujishige [1987] has extended the same results for an arbitrary submodular system and has presented an algroithm to get it. His algorithm, which is not a direct extension of the algorithm for polymatroid, contains an oracle computation which has been pointed out by G. Morton, R. von Randow and K. Ringwald [1985]. Here, we show a greedy procedure to get it through algebraic consideration, which is quite different from Fujishige's algorithm $[1980,1987)$, but is an algebraic counterpart of his geometric consideration.

Submodular system is essentially a poset greedoid with submodular funclion on it, which is implicilly stated in S. Fujishige and N. Tomizawa [1983]. Greedoids are crealed and has been investigated by B. Korte and L. Lovás: [1982-1936]. Our result is a nalural consequence through the study of greedoids and submodular systems. 
2. Submodular system, submodular polyhedra and their basic characteristics

We use the same symbol and terminology as that of S. Fujishige [1984]. Let $E$ be a finite set and denote by $2^{E}$ the set of all the subsets of $E$. Let a collection $\mathcal{D}$ of subsets of $E$ be a distributive lattice with set union and intersection as the lattice operations, i.e., for any $X, Y \in \mathcal{D}$ we have $X \cup Y, X \cap Y \in \mathcal{D}$. A function $f$ from $\mathcal{D}$ to the set $R$ of reals is called a submodular function on $\mathcal{D}$ if for each pair of $X, Y \in \mathcal{D}$

$$
f(X)+f(Y) \geq f(X \cup Y)+f(X \cap Y)
$$

A pair $(\mathcal{D}, f)$ of a distributive lattice $\mathcal{D} \subseteq 2^{E}$ and a submodular function $f: \mathcal{D} \rightarrow R$ is called a submodular system. We assume that $\emptyset, E \in \mathcal{D}$ and $f(\theta)=0$. Note that the value $f(\theta)$ doesn't affect the other value $f(A)$ at $A \in D$ because $A \cup \emptyset=A, A \cap \emptyset=\emptyset$. Given a submodular system $(\mathcal{D}, f)$, define a polyhedron $P$, by

$$
P_{f}:=\left\{x \in R^{E} \mid x(X) \leq f(X)(\forall X \in \mathcal{D})\right\}
$$

where $R^{E}$ is the set of vectors $x=(x(e): e \in E)$ with coordinates indexed by $E$ and $x(e) \in R(e \in E)$ and

$$
x(X):=\sum_{e \in X} x(e)
$$

We call $P_{f}$ the submodular polyhedron associated with the submodular system $(D, f)$. Define

$$
B_{f}:=\left\{x \in P_{f} \mid x(E)=f(E)\right\} \text {, }
$$

which is called the base polyhedron associated with $(\mathcal{D}, f)$.

Lemma 2.1 Let $x \in P_{f}$ and $A, B \in \mathcal{D}$. If $x(A)=f(A), x(B)=f(B)$, then $x(A \cap B)=f(A \cap B)$ and $x(A \cup B)=f(A \cup B)$ hold.

Prool. Same as that of S. Fujishige [1978].

Let $\chi_{u}$ be a characteristic funclion of $u$, i.e., $\chi_{u}(e)=1$ for $e=u$ and $\chi_{u}(e)=0$ for $e \in E \backslash\{u\}$. Define a saturation function sat ()$: P_{f} \rightarrow 2^{E}$ by $\operatorname{sat}(x):=\left\{u \in E \mid \forall d>0, x+d \chi_{u} \notin P_{f}\right\}\left(x \in P_{f}\right)$. Then we have the following lemma, where $\wp(x):=\{A \in \mathcal{D} \mid x(A)=f(A)\}$. 
Lemuna 2.2 Let $x \in P_{f}$. Then $\operatorname{sat}(x)$ satisfies

$$
\operatorname{sat}(x) \in \mathcal{D}, x(\operatorname{sat}(x))=f(\operatorname{sat}(x))
$$

Furthermore, $p(x)$ is a distributive lattice with a partial order relation defined by the set inclusion and sat $(x)$ is the maximum element of $p(x)$.

Proof. Same as that of S. Fujishige [1980].

Note that $\operatorname{sat}(x)$ is a function from $P_{f}$ into $\mathcal{D}$.

Lemuna 2.3 Let $x \in P_{f}$. Then $x \in B_{f}$ iff $\operatorname{sat}(x)=E$.

Proof. Use the definition of $B_{f}$ and Lemma 2.2 .

For $x \in P_{f}, u \in \operatorname{sat}(x)$, we can define dependence function $\operatorname{dep}(): P_{f} \rightarrow \mathcal{D}$ and also we can introduce capacity, exchande capacity and so on (Fujishige $(1984,1987])$, but we don't go into the details because we don't use them.

Let $n:=|E|$. For any real sequences $a=\left(a_{1}, \ldots, a_{n}\right)$ and $b=\left(b_{1}, \ldots, b_{n}\right)$ of length $n, a$ is called lexicographically greater than or equal to $b$ if for some $j \in\{1, \ldots, n\}$.

$$
\begin{aligned}
& a_{i}=b_{i} \quad(i=1, \ldots, j-1) \\
& a_{j}>b_{j} \\
& \text { or } \\
& a_{i}=b_{i}(i=1, \ldots, n) .
\end{aligned}
$$

A vector $w \in R^{E}$ such that $w(e)>0(e \in E)$ is called a weight vector. For a vector $x \in R^{E}$, denote by $T(x)$ the $n$-tuple (or sequence) of the numbers $x(e)(e \in E)$ arranged in order of increasing magnitude. Given a weight vector $w$, a base $x$ of $(\mathcal{D}, f)$ is called a lexicographically optimal base with respect to the weight vector $w$ if the $n$-tuple $T\left((x(e) / w(e))_{e \in E}\right)$ is lexicographically maximum among all $n$-tuples $T\left((y(e) / w(e))_{e \in E}\right)$ for all bases $y$ of $(D, f)$. The mathematical Programming problem to get $x \in B$, such that

$$
T\left((x(e) / w(e))_{e \in E}\right)=\begin{gathered}
\text { Lexicographically maximum } \\
\text { subject to } y \in B_{f}
\end{gathered} T\left((y(e) / w(e))_{e \in E}\right)
$$

is called wlob (weighted lexicographically optimal base) problem for submodular system. 
3. Existence and uniqueness of a lexicographically optimal base with respect to a weight vector

Let $c_{1}:=\min \left\{\frac{\int(A)}{w(A)} \mid \emptyset \neq A \in \mathcal{D}\right\}, u_{c_{1}}(e):=c_{1} w(e)(e \in E)$. Then we see that $u_{c_{1}} \in P_{f}$ holds. By Lemma 2.2, we have $u_{c_{1}}\left(\operatorname{sat}\left(u_{c_{1}}\right)\right)=f\left(\operatorname{sat}\left(u_{c_{1}}\right)\right)$. Let $A_{1}$ be a set such that $\left.c_{1}=\int\left(A_{1}\right), \emptyset \neq A_{1}\right), \mathcal{D}$. Then $A_{1} \subseteq \operatorname{sat}\left(u_{c_{1}}\right)$, because $\forall e \in A_{1}, \forall d>0,\left(u_{c_{1}}+d \chi_{e}\right)\left(A_{1}\right)=c_{1} w\left(A_{1}\right)+d>f\left(A_{1}\right)$. Thus we get $\emptyset \neq \operatorname{sat}\left(u_{c_{1}}\right) \in \mathcal{D}$. Therefore, we are in a position such that

$u_{c_{1}}(e)=c_{1} w(e)(e \in E), u_{c_{1}} \in P_{f}, \emptyset \neq \operatorname{sat}\left(u_{c_{1}}\right) \in \mathcal{D}$ and $u_{c_{1}}\left(\operatorname{sat}\left(u_{c_{1}}\right)\right)=f\left(\operatorname{sat}\left(u_{c_{1}}\right)\right)$.

In case $\operatorname{sat}\left(u_{c_{1}}\right)=E$, by Lemma 2.3 , we see that

$$
u_{c_{1}} \in B_{f} . \text { STOP }
$$

In case $\operatorname{sat}\left(u_{c_{1}}\right) \notin E$, let $\epsilon_{1}:=\min \left\{\frac{f(A)-u_{c_{2}}(A)}{\left.w(A) \backslash \operatorname{sat}\left(u_{c_{1}}\right)\right)} \mid A \backslash \operatorname{sat}\left(u_{c_{1}}\right) \neq D, A \in \mathcal{D}\right\}$. Then by Lemma 2.1, we get $\epsilon_{1}>0$. Let $c_{2}:=c_{1}+\epsilon_{1}$, and let

$$
u_{c_{2}}(e):= \begin{cases}c_{1} w(e)=u_{c_{1}}(e) & \text { for } e \in \operatorname{sat}\left(u_{c_{1}}\right) \\ c_{2} w(e)=u_{c_{1}}(e)+\epsilon_{1} w(e) & \text { for } e \in E \backslash \operatorname{sat}\left(u_{c_{1}}\right) .\end{cases}
$$

By the definition of $u_{c_{2}}$ and $\epsilon_{1}$, and by the fact that $u_{c_{1}} \in P_{f_{f}}$, we get $u_{c_{1}} \in$ $P_{f}$. Furthermore we get $\mathscr{p}\left(u_{c_{1}}\right) \subseteq \wp\left(u_{c_{2}}\right)$ and so $\operatorname{sal}\left(u_{c_{1}}\right) \subseteq \operatorname{sat}\left(u_{c_{2}}\right)$. From the definition of $\epsilon_{1}$, we have a set $A_{1} \in \mathcal{D}, A_{1} \backslash \operatorname{sat}\left(u_{c_{1}}\right) \neq \emptyset$ such that $\epsilon_{1}=$ $\frac{f\left(A_{1}\right)-u_{c_{1}}\left(A_{1}\right)}{w\left(A_{1} \backslash \operatorname{sat}\left(u_{c_{1}}\right)\right)}$. Then

$$
\begin{aligned}
u_{c_{2}}\left(A_{1}\right) & =u_{c_{2}}\left(A_{1} \cap \operatorname{sat}\left(u_{c_{1}}\right)\right)+u_{c_{2}}\left(A_{1} \backslash \operatorname{sat}\left(u_{c_{1}}\right)\right) \\
& =c_{1} w\left(A \cap \operatorname{sat}\left(u_{c_{1}}\right)\right)+\left(c_{1}+\epsilon_{1}\right) w\left(A_{1} \backslash \operatorname{sat}\left(u_{c_{1}}\right)\right)\left[\text { by the definition of } u_{c_{2}}\right] \\
& =c_{1} w\left(A_{1}\right)+\epsilon_{1} w\left(A_{1} \backslash \operatorname{sat}\left(u_{c_{1}}\right)\right)=u_{c_{1}}\left(A_{1}\right)+\epsilon_{1} w\left(A_{1} \backslash \operatorname{sat}\left(u_{c_{1}}\right)\right)=f\left(A_{1}\right)
\end{aligned}
$$

and so $A_{1} \in \mathfrak{p}\left(u_{c_{2}}\right)$.

By Lemma 2.1 and $\operatorname{sat}\left(u_{c_{1}}\right) \in \mathfrak{p}\left(u_{c_{2}}\right)$, we have sat $\left(u_{c_{1}}\right) \nsubseteq^{*} \operatorname{sat}\left(u_{c_{1}}\right) \cup A \in$ $p\left(u_{c_{2}}\right)$. Thus sat $\left(u_{c_{1}}\right) \notin \operatorname{sat}\left(u_{c_{2}}\right)$. From Lemma 2.2 and $u_{c_{2}} \in P_{f}$, we have

$$
u_{c_{2}}\left(\operatorname{sat}\left(u_{c_{2}}\right)\right)=f\left(\operatorname{sat}\left(u_{c_{2}}\right)\right) \text {. }
$$

Therefore, we are in a position such that

$u_{c_{3}}(e)=\left\{\begin{array}{l}c_{1} w(e)\left(e \in \operatorname{sal}\left(u_{c_{1}}\right)\right) \\ c_{2} w(e)\left(e \in E \backslash \operatorname{sat}\left(u_{c_{1}}\right)\right), \quad u_{c_{i}} \in P_{J}(i=1,2), 0 \neq \operatorname{sal}\left(u_{c_{1}}\right) Z \operatorname{sal}\left(u_{c_{2}}\right) \subseteq \mathcal{D},\end{array}\right.$

$u_{c_{i}}\left(\operatorname{sat}\left(u_{c_{i}}\right)\right)=f\left(\operatorname{sat}\left(u_{c_{i}}\right)\right)(1 \leq i \leq 2)$ and $c_{1}<c_{2}$.

- $X \subseteq Y$ means that $X$ is a proper subset of $Y$. 
Continuing this process, we get $u_{c_{p}}$ such that $\operatorname{sat}\left(u_{c_{p}}\right)=E$, i.e., $u_{c_{p}} \in B_{f}$. Set

$$
c(e):=\left\{\begin{array}{l}
c_{1}\left(e \in \operatorname{sat}\left(u_{c_{1}}\right)\right) \\
c_{2}\left(e \in \operatorname{sat}\left(u_{c_{1}}\right) \backslash \operatorname{sat}\left(u_{c_{1}}\right)\right) \\
\vdots \\
c_{i}\left(e \in \operatorname{sat}\left(u_{c_{i}}\right) \backslash \operatorname{sat}\left(u_{c_{-1}}\right)\right) \\
\vdots \\
c_{p}\left(e \in \operatorname{sat}\left(u_{c_{p}}\right) \backslash \operatorname{sat}\left(u_{c_{p-1}}\right)=E \backslash \operatorname{sat}\left(u_{c_{p-1}}\right) .\right.
\end{array}\right\}
$$

Then we have

$$
u_{c_{p}}(e)=\left\{\begin{array}{l}
c_{1} w(e)\left(e \in \operatorname{sat}\left(u_{c_{1}}\right)\right) \\
c_{2} w(e)\left(e \in \operatorname{sat}\left(u_{c_{2}}\right) \backslash \operatorname{sat}\left(u_{c_{1}}\right)\right) \\
\vdots \\
c_{i} w(e)\left(e \in \operatorname{sat}\left(u_{c_{i}}\right) \backslash \operatorname{sat}\left(u_{c_{i-1}}\right)\right) \\
\vdots \\
c_{p} w(e)\left(e \in \operatorname{sat}\left(u_{c_{p}}\right) \backslash \operatorname{sat}\left(u_{c_{p-1}}\right)\right)
\end{array}\right.
$$

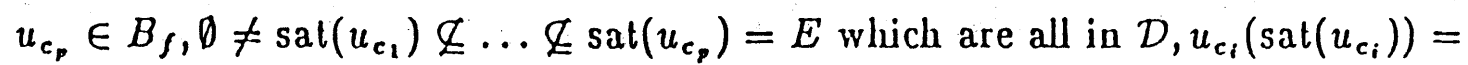
$f\left(\operatorname{sat}\left(u_{c_{i}}\right)\right)(1 \leq i \leq p)$ and

$$
c_{1}<\ldots<c_{p}
$$

Note. For a positive submodular system $(\mathcal{D}, f)$, i.e., submodular system with $f(A)>0(\emptyset \neq A \in \mathcal{D})$, we see that $c_{1}>0$.

Theorem 3.1 (Existence) Let $c(e)(e \in E)$ be those defined by (3.4). Then the vector $x$ defined by

$$
x=(c(e) w(e))_{e \in E}
$$

is a lexicographically optimal base with respect to the weight vector $w$.

Proof. Let $z \in B_{f}$. We show that

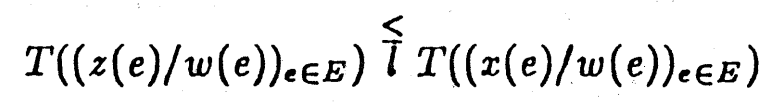

holds. First note that

$$
z(A) \leq f(A) \quad(\emptyset \neq A \in \mathcal{D})
$$

holds. Let $q:=\left(q_{1}, \ldots, q_{n}\right), n=|E|$, be any permutation corresponding to $x$ such that

$$
\frac{x\left(q_{1}\right)}{w\left(q_{1}\right)}=\ldots=\frac{x\left(q_{j_{1}}\right)}{w\left(q_{j_{1}}\right)}=c_{1}<\frac{x\left(q_{j_{1}+1}\right)}{w\left(q_{j_{1}+1}\right)}=\ldots=\frac{x\left(q_{j_{2}}\right)}{w\left(q_{j_{2}}\right)}=c_{2}<\ldots<
$$


$\frac{x\left(q_{j_{p}-1+1}\right)}{w\left(q_{j_{p}-1+1}\right)}=\ldots=\frac{x\left(q_{j_{p}}\right)}{w\left(q_{j_{p}}\right)}=c_{p}, j_{p}=n, c_{j_{0}}=0$. Let $S_{i}=\left\{q_{j_{i-1}+1}, q_{j_{i-1}+2}, \ldots, q_{j_{i}}\right\}(1 \leq$ $i \leq p)$. Then we have $S_{1}=\operatorname{sat}\left(u_{c_{1}}\right), S_{i}=\operatorname{sat}\left(u_{c_{i}}\right) \backslash \operatorname{sat}\left(u_{c_{i-1}}\right)(2 \leq i \leq p)$.

If $\frac{x\left(q_{1}\right)}{w\left(q_{1}\right)}<c_{1}$, then $(3.7)$ holds.

If $\frac{z\left(q_{1}\right)}{w\left(q_{1}\right)} \geq c_{1}, \frac{z\left(q_{2}\right)}{w\left(q_{2}\right)}<c_{1}$, the $(3.7)$ holds.

:

If $\frac{2\left(q_{1}\right)}{w\left(q_{1}\right)} \geq c_{1}, \ldots, \frac{2\left(q_{i_{1}}\right)}{w\left(q_{j_{1}}\right)} \geq c_{1}$, then we see that

$$
\frac{z(e)}{w(e)}=\frac{x(e)}{w(e)}=c_{1}\left(e \in S_{1}\right)
$$

holds by $z\left(S_{1}\right) \geq c_{1} w\left(S_{1}\right)=u_{c_{1}}\left(S_{1}\right)=f\left(S_{1}\right)$ and by $(3.8)$.

If $\frac{z(e)}{w(e)}=c_{1}\left(e \in S_{1}\right) ; \frac{z\left(q_{j_{1}+1}\right)}{w\left(\eta_{\left.j_{1}+1\right)}\right.}<c_{2}$, then (3.7) holds.

If $\frac{z(e)}{w(e)}=c_{1}\left(e \in S_{1}\right), \frac{z\left(q_{j+1}\right)}{w\left(q_{j_{1}+1}\right)} \geq c_{2}, \frac{z\left(q_{j 1}+2\right)}{w\left(q_{j_{1}}+2\right)}<c_{2}$, then (3.7) holds.

$\vdots$

If $\frac{z(e)}{w(e)}=c_{1}\left(e \in S_{1}\right), \frac{x\left(q_{j_{1}+1}\right)}{w\left(q_{j_{1}+1}\right)} \geq c_{2}, \ldots, \frac{z\left(q_{j_{2}}\right)}{w\left(q_{j_{2}}\right)} \geq c_{2}$, then we see that $\frac{x(e)}{w(e)}=$ $c_{2}=\frac{x(e)}{w(e)}\left(e \in S_{2}\right)$ holds because $z(e)=c_{1} w(e)\left(e \in S_{1}\right)$ and $z\left(S_{2}+S_{1}\right) \leq$ $f\left(S_{2}+S_{1}\right), f\left(S_{2}+S_{1}\right)=u_{c_{2}}\left(S_{2}+S_{1}\right)=z\left(S_{1}\right)+c_{2} w\left(S_{2}\right) \leq z\left(S_{2}+S_{1}\right)$. Continuing in this way, we see that (3.7) holds for any $z \in B_{f}$.

Theorem 3.2 (Uniqueness, Fujishige, S. (1980]) Let $c(e)(e \in E)$ be those defined by (3.4). Then the vector $x$ defined by (3.6) is the unique lexicographically optimal base of $(\mathcal{D}, f)$ with respect to a weight vector $w$.

Proof. Same as that of Fujishige, S. [1980]. Use (3.5), especially sat $\left(u_{c_{i}}\right) \in$ $\mathcal{D}, u_{c_{i}}\left(\operatorname{sat}\left(u_{c_{i}}\right)\right)=f\left(\operatorname{sat}\left(u_{c_{i}}\right)\right)$.

Based on these algebraic arguments, we present an algorithm to get the lexicographically optimal base of a submodular system $(D, f)$ with respect to a weight vector $w$.

Algorithm to get the lexicographically optimal base

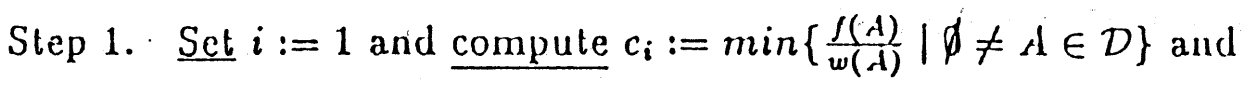
set $u_{c_{i}}(e):=c_{i} w(e)(e \in E)$.

Step 2. If $\operatorname{sat}\left(u_{c_{i}}\right)=E$, then $\operatorname{STOP}$. 
Step 3. Compute $\epsilon_{i}:=\min \left\{\frac{f(A)-u_{c_{i}}(A)}{w\left(A \backslash \operatorname{sat}\left(u_{c_{i}}\right)\right)} \mid A \in \mathcal{D}, A \backslash \operatorname{sat}\left(u_{c_{i}}\right) \neq \emptyset\right\}$

and $\underline{\text { set }} c_{i+1}:=c_{i}+\epsilon_{i}$ and set

$u_{c_{i+1}}(e):= \begin{cases}u_{c_{i}}(e) & \text { for } e \in \operatorname{sat}\left(u_{c_{i}}\right) \\ u_{c_{i}}(e)+\epsilon_{i} w(e) & \text { for } e \in E \backslash \operatorname{sat}\left(u_{c_{i}}\right) .\end{cases}$

Set $i:=i+1$ and go to Step 2 .

Theorem 3.3 (Fujishige, S. [1980]) Let $\hat{x} \in B_{f}$ and let $w$ be a weight vector. Define

$$
\hat{c}(e):=\hat{x}(e) / w(e)(e \in E)
$$

and let the distinct numbers of $\hat{c}(e)(e \in E)$ be given by

$$
\hat{c_{1}}<\hat{c_{2}}<\ldots<\hat{c}_{\hat{p}}
$$

Furthermore, define $\hat{S}_{\mathbf{i}} \subseteq E(1 \leq i \leq \hat{p})$ by $\hat{S}_{\boldsymbol{i}}:=\left\{e \in E \mid \hat{c}(e) \leq \hat{c}_{i}\right\}(1 \leq i \leq \hat{p})$. Then the following three conditions are equivalent:

(i) $\hat{x}$ is the lexicographically optimal base of $P_{f}$ with respect to $w$;

(ii) $\hat{S}_{i} \in \mathcal{D}$ and $\hat{x}\left(\hat{S}_{i}\right)=f\left(\hat{S}_{i}\right)(1 \leq i \leq \hat{p})$;

(iii) For any $e \in \hat{S}_{i}, \emptyset \neq \operatorname{dep}(\hat{x}, e) \subseteq \hat{S}_{i}(1 \leq i \leq \hat{p})$.

Remark If one of the three conditions holds, then we have $\hat{p}=p$.

Given a submodular system $(\mathcal{D}, f)$ and a weight vector $w$ and $p>1$, define a mathematical programming problem

$$
\mathrm{P} \quad: \text { minimize } f_{w}(x)=\frac{1}{p} \sum_{e \in E} \frac{x(e)^{p}}{w(e)^{p-1}} \text { subject to } x \in B_{f} \text { and } x \geq 0 \text {. }
$$

Fujishige, S. [1980]showed that for a polymatroid $(\mathcal{D}, f)$ with $p=2$, its unique solution is the lexicograhphically optimal base w.r.t. $w$. Morton, G. and von Randow, R. and Ringwald, K. [1985] extended it for $p>1$, where $(\mathcal{D}, f)$ is a polymatroid. We can easily see that for a positive submodular system $(\mathcal{D}, f)$ with $p>1$, the same result holds. As for an arbitrary submodular system, $F$ might be infeasible. For example, for a submodular system $(\mathcal{D}, f)$ with $f(A)<$ $0(A \in \mathcal{D})$. So, consider another problem

$$
\hat{P}: \text { minimize } f_{w}(x)=\frac{1}{p} \sum_{e \in E} \frac{x(e)^{p}}{w(e)^{p-1}} \text { subject to } x \in B_{f} .
$$


We have an example for which $\hat{P}$ has no optimal solution as follows: Let $E=$ $\{1,2,3\}, \mathcal{D}=\{\emptyset,\{3\},\{1,2,3\}\}, f(\emptyset)=0, f(\{3\})=-2, f(\{1,2,3\})=-3$. Then $(\mathcal{D}, f)$ is a submodutar system with base polyhedron $B_{f}=\left\{\left(x_{1}, x_{2}, x_{3}\right) \mid x_{1}+\right.$ $\left.x_{2}+x_{3}=-3, x_{3} \leq-2\right\}$. Let $w=(1,1,1)$. The lexicographically optimal base $x^{*}$ becomes $x^{*}=\left(-\frac{1}{2},-\frac{1}{2},-2\right)$. Let $p=3$ and let $x_{1}=x_{2}=-\frac{(t+3)}{2}, x_{3}=t(\leq-2)$. Then $\left(x_{1}, x_{2}, x_{3}\right) \in B_{f}$ with $3 f_{w}(x)=t^{3}-\frac{1}{4}(t+3)^{3} \rightarrow-\infty$ as $t \rightarrow \infty$. Problem $\hat{P}$ for this case has no minimum solution. For an even natural number $p$, if there exists a minimum solution $\hat{x}$ for $\hat{P}$, then we see that $\hat{x}$ is the lexicographically optimal base w.r.t. $w$.

Theorem 3.4 (Fujishige, S. [1980], Morton, G. and von Randow, R. and Ringwald, K. [1985])

Let $x^{*}$ be the lexicographically optimal base of a positive submodular system $(\mathcal{D}, f)$ with respect to a weight vector $w$ and let $p>1$. Then $x^{*}$ is the unique optimal solution of the problem $\mathbf{p}$.

\section{Example}

We will show here that the first problem of G. Morton, R. von Randow and K. Ringwald [1985]can be solved within our framework. Their problem is as follows:

$$
\min \sum_{j=1}^{n} \lambda_{j} x_{j}^{p} \text { subject to } A x \geq c, x \geq 0,
$$

where $\lambda_{j}>0(1 \leq j \leq n), p>1, c_{n} \geq c_{n-1} \geq \ldots \geq c_{1} \geq 0$, and

$$
A=\left(a_{i j}\right)_{n \times n} \text { with } a_{i j}= \begin{cases}1, & i \geq j \\ 0, & i<j\end{cases}
$$

Let $e_{i}$ be the $i$-th column vector of $A, E:=\left\{e_{i} \mid 1 \leq i \leq n\right\}, F_{j}:=\left\{e_{i} \mid\right.$ $1 \leq i \leq j\}(1 \leq j \leq n), F_{0}:=\emptyset, D_{j}:=E \backslash F_{j}=\left\{e_{j+1}, \ldots, e_{n}\right\}(0 \leq j \leq n)$. Let $\mathcal{D}=\left\{E=D_{0}, D_{1}, \ldots, D_{n-1}, D_{n}=\emptyset\right\}$. Let $\rho\left(D_{j}\right):=c_{n}-c_{j}(0 \leq j . \leq n)$, where $c_{0}=0$. Then $(E, \mathcal{D}, \rho)$ is a submodular system with $\emptyset, E \in \mathcal{D}, \rho(\emptyset)=0$. For $x, y \in \mathbf{R}_{+}^{n}$, define $x \leq y$ if $x(e) \leq y(e)(e \in E)$, where $\mathbb{R}_{+}$is the set of nonnegative reals. $\left(\mathbb{R}_{+}^{n}, \leq\right)$ is a poset with this partial order. Define $P:=\{x \in$ $\left.\mathbb{R}_{+}^{n} \mid A x \geq c\right\}, O(4.1):=$ the set of optimal solutions to (4.1), minimal $P:=$ the set of minimal elements of $P$. Then we easily see that

$$
O(4.1) \subseteq B_{p} \subseteq \text { minimal } P \subseteq P
$$

Hence problem (4.1) is equivalent to

$$
\min \left\{\frac{1}{p} \sum_{i=1}^{n} x\left(e_{i}\right)^{p} w\left(e_{l}\right)^{1-p} \mid x \in B_{p}\right\} \text {, }
$$


where $w\left(e_{i}\right)=\lambda_{i}^{-\frac{1}{(-1)}}$. Let $d_{j}=\sum_{i=1}^{j} w\left(e_{i}\right)(1 \leq j \leq n)$ and $d_{0}=0$. Then $w\left(D_{j}\right)=d_{n}-d_{j}(0 \leq j \leq n)$. Apply our algorithm to this problem:

$c_{1}^{\prime}:=\min \left\{\frac{\rho\left(D_{j}\right)}{w\left(D_{j}\right)} \mid 0 \leq j \leq n-1\right\}=\min \left\{\frac{c_{n}-c_{0}}{d_{n}-d_{0}}, \frac{c_{n}-c_{1}}{d_{n}-d_{1}}, \frac{c_{n}-c_{2}}{d_{n}-d_{2}}, \ldots, \frac{c_{n}-c_{n-1}}{d_{n}-d_{n-1}}\right\}$

Let $s^{\prime}(0)=n$ and $c_{1}^{\prime}=\frac{c_{n}-c_{1}(1)}{d_{n}-d_{,}(1)}$ and $u_{c_{1}^{\prime}}\left(e_{i}\right)=c_{1}^{\prime} w\left(e_{i}\right)(1 \leq i \leq n)$. Then $u_{c_{1}^{\prime}}\left(D_{j}\right)=c_{1}^{\prime}\left(d_{n}-d_{j}\right), \operatorname{sat}\left(u_{c_{1}^{\prime}}\right)=\bigcup\left\{A \mid A \in \mathcal{D}, u_{c_{1}^{\prime}}(A)=\rho(A)\right\}=D_{s^{\prime}(1)}$ for which $s^{\prime}(1)$ is the least index $j$ such that $c_{1}^{\prime}=\frac{c_{n}-c_{j}}{d_{n}-d_{j}}, 0 \leq s^{\prime}(1)<s^{\prime}(0)$.

If $s^{\prime}(1)=0$, then $\operatorname{sat}\left(u_{c_{i}^{\prime}}\right)=E$. STOP.

If $s^{\prime}(1) \neq 0$, then $\operatorname{sat}\left(u_{c_{1}^{\prime}}\right) \neq E$ and so compute

$\dot{\epsilon}_{1}^{\prime}:=\min \left\{\frac{\rho(A)-u_{c_{1}^{\prime}}(A)}{w\left(A \backslash \operatorname{sat}\left(u_{c_{1}^{\prime}}\right)\right)} \mid A \in \mathcal{D}, A \backslash \operatorname{sat}\left(u_{c_{1}^{\prime}}\right) \neq \emptyset\right\}=\min \left\{\frac{c_{n}-c_{j}-c_{1}^{\prime}\left(d_{n}-d_{j}\right)}{d_{s^{\prime}(1)}-d_{j}} \mid\right.$

$\left.0 \leq j \leq n-1, j<s^{\prime}(1)\right\}$, where $\frac{c_{n}-c_{j}-c_{1}^{\prime}\left(d_{n}-d_{j}\right)}{d_{s^{\prime}(1)}-d_{j}}=\frac{c_{g^{\prime}(1)}-c_{j}}{d_{s^{\prime}(1)}-d_{j}}-c_{1}^{\prime}$.

Let $\epsilon_{1}^{\prime}:=\frac{c_{f^{\prime}(1)}-c_{g^{\prime}(1)}}{d_{\rho^{\prime}(1)}-d_{\rho^{\prime}(1)}}-c_{1}^{\prime}$. Then $\left(d_{s^{\prime}(2)}, c_{s^{\prime}(2)}\right)$ is a point $\left(d_{j}, c_{j}\right), 0 \leq j<s^{\prime}(1)$ with the smallest slope coefficient $\frac{c_{1}^{\prime}(1)-c_{j}}{d_{\theta^{\prime}(1)}-d_{j}}$. Hence we see that

$$
s^{\prime}(0)=n=s(m), s^{\prime}(1)=s(m-1), \ldots, s^{\prime}(m-1)=s(1), s^{\prime}(m)=s(0),
$$

which is the same result as that of G. Morton, R. von Randow and K. Ringwald, although the decision proceeds inversely. The reader would have noticed that the $(E, D)$ here, is a poset greedoid which comes from a chain as follows:

$$
\begin{aligned}
& \left\{\begin{array}{l}
e_{1} \\
e_{2}
\end{array}\right. \\
& \vdots \vdots \\
& \left\{\begin{array}{l}
e_{n-1} \\
e_{n}
\end{array}\right.
\end{aligned}
$$

The reason for the in $r e r s e$ decision process will be investigaled in anolher paper. 
Kakuzo Iwamura

\section{References}

Björner, A. [1983): On matroids, groups and exchange languages. Colloquia Mathematica Societatis Janós Bolyai vol. 40 (1985), pp. $25^{\circ}-60$.

Björner, A. and Korte, B. and Lorass, L. (1985): Homotopy properties of greedoids. Advances in Applied Mathematics 6 (1985), ipp. 447-494.

Fujishige, S. [1978]: Algorithms for solving the independent-now probleni. J. of the Operations Research Society of Japan, vol. 21, no. 2 (1978), p. 189-202.

Fujishige, S. [1980]: Lexicographically optimal base of a polymatroid with respect to a weight vector. Math. of O.R., vol. 5, no. 2 (1980), pp. 186-19u.

Fujishige, S. and Tomizawa, N. [1983]: A note on submodular functions on distribut ive lattices. J. of the Operations Research Society of Japan, vol. 26, no. 4 (1983), pp. 309-317.

Fujishige, S. [1984]: Submodular systems and related topics. Mathematical Programming Study 22 (1984), pp. 113-131.

Fujishige, S. [1987]: private communication.

[wamura, K. and Deguchi, Y. and Nakayama, T. (198T): A greedy procedure to get a lexicographically optimal base of a positive submodular system with respect to a weight vector. working paper.

Korte, B. and Lovász, L. [1982]: Posets, matroids and greedoids. in, Colloquia Mathematica Societatis Janós Bolyai 40 (1982), pp. 239-265.

Korte, B. and Lovász, L. [1983]: Structural properties of greedoids. Combinatorica 3 (3-4) (1983), pp. 359-374.

Korte, B. and Lovász, L. (1984a): Greedoids - a structural framework for the greedy algorithm. in: W.R. Pulleyblank (ed.): Progress in Combinatorial Optimization, Academic Press, 1984, pp. 221-243.

Korte, B. and Lorász, L. [1984b]: Shelling structures, convexity and a happy end. in: $B$. Bollobás (ed.): Graph Theory and Combinatorics, Academic Piess (1984), pp. 219-232.

Korte, B. and Lovász, L. [1984c]: Greedoids and linear objective functions. SIANI J. ALG. DISC. Math. vol. 5, no. 2 (1984), pp. 229-238.

Korte, B. and Luvisz, L. (1985j: Polymatruid grcedoid. J. of Combinatorial Theory, Serics B, vol. 38, no. 1 (1985), pp. \$1-72.

Korte, B. and Lovász, L. [1935): A note on selectors and grfedoids. Europ. J. of Combinatorics 6 (1985), pp. 59-67. 
Lexicograplically optimal base of a submodular system

Korte. B. and Lovász, L. (1986): Homomorphisms and Ramsey properties of antimatroids. Discrete Applied Mathematics 15 (1986), pp. 283-290.

Morton, G. and ron Randow, R. and Ringwald, K. [1985]: $\Lambda$ greedy algorithm for solving a class of convex programming problems and its connection with polymatroid theory. Math. Program. 32 (1985), pp. 238-241. 